\title{
Mesures \\ des gradients thermiques dans l'intercuve du réacteur Phénix
}

\author{
par J. Branchu \\ G.A.A.A.
}

\author{
ef J.M. Gama \\ Technicatome
}

\section{Introduction}

Pour le réacteur surrégénérateur Phénix $(250 \mathrm{MWe})$ les répartitions de températures, pendant les différents régimes de fonctionnement et les transitoires, sur les structures internes immergées dans le sodium primaire sont importantes pour vérifier la bonne tenue de ces structures sous l'effet des contraintes thermiques qui peuvent en découler.

Des estimations de ces répartitions de température ont été faites au cours des études. Elles ont permis de prendre un certain nombre de décisions concernant le dessin des structures internes.

Des mesures de ces températures ont été effectuées, grâce à l'instrumentation en thermocouples placés dans le sodium primaire, à différents régimes de fonctionnement depuis le début des essais de montée en puissance de la centrale (30-10-1973) jusqu'à maintenant.

Cet exposé donne les résultats de ces mesures actuellement disponibles.

\section{1 - Description sommaire du bloc réacteur}

(Voir fig. 1 et 2)

Le bloc réacteur comporte une cuve principale dans laquelle est intégré l'ensemble du «circuit» de sodium primaire dont les principaux composants sont (dans le sens de circulation du fluide caloporteur):

- les assemblages combustibles et fertiles parcourus par le sodium de bas en haut;

- le couvercle-cœur portant l'instrumentation de sortie des assemblages;

- le collecteur chaud à l'intérieur de la cuve primaire;
- la cuve primaire séparant le sodium primaire chaud du sodium primaire froid;

- les six échangeurs intermédiaires parcourus du haut en bas par le sodium primaire, à l'extérieur du faisceau de tubes, qui traversent la cuve primaire; les fenêtres d'entrée du sodium chaud sont protégées par les cloches d'entrée suspendues au toit de la cuve; les fenêtres de sortie rejettent le sodium primaire refroidi dans l'espace annulaire, dénommé «intercuve», délimité intérieurement par la virole inférieure et le redan de la cuve primaire et extérieurement par la cuve principale, protégée par son baffle, et la virole tronconique support du platelage;

- les trois jupes d'aspination du sodium froid par lesquelles les trois pompes primaires sont alimentées en sodium froid du bas de l'intercuve;

- les trois pompes primaires refoulent le sodium froid par trois tubulures dans une chambre cylindrique, appelée «sommier» support des assemblages;

- le sommier assure l'alimentation des assemblages au débit correspondant à leur position dans le cœur du réacteur;

- une partie du sodium froid, entrant dans le sommier, fuit au niveau du pied des assemblages vers le fond de la cuve principale; cette fuite est utilisée pour maintenir froide la cuve principale par une circulation ménagée entre la paroi de la cuve principale et le baffle, de bas en haut; cette circulation rebrousse chemin vers le bas près de la surface libre et est canalisée entre le baffle et un contrebaffle, pour être finalement rejetée dans l'intercuve.

La figure 1 montre une coupe verticale du bloc réacteur par une pompe et un échangeur.

La figure 2 est la coupe horizontale du bloc réacteur au niveau des têtes d'assemblages. 


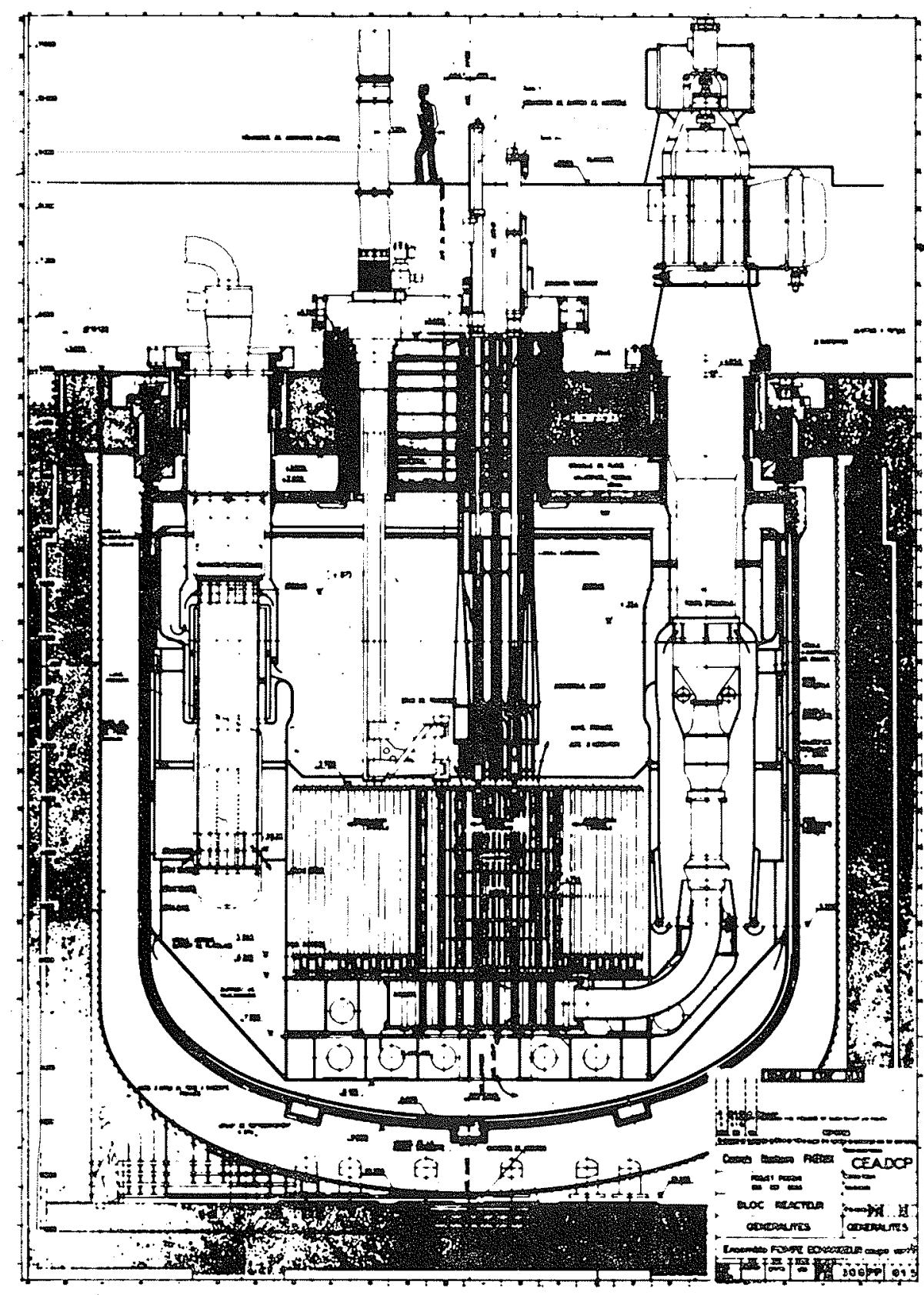




\section{2 - Instrumentation}

\section{en thermocouples des structures immergées en sodium}

Des thermocouples Chromel-Alumel en câble Pyrotenax ont été installés sur les différentes structures du bloc réiacteur: - Cuve primaire .......... :26 thermocouples

- Perche entre protection neutronique latérale et cuve primaire ....

8 thermocouples

- Perche dintercuve ..........

19 thermocouples

- Baffle et contrebaffle ........

5 thermocouples

- Jupe de pompe ...........

8 thermocouples

Soit 66 thermocouples immergés en sodium dans la zone de l'intercuve et sur les structures internes.

Il faut noter que, par souci d'éviter qu'au cours de la vie du réacteur, certains de ces thermocouples n'en viennent à se corroder, à se détacher et à constituer des débris susceptibles d'être entraînés par le courant de sodium, toute cette instrumentation (fixation de la soudure chaude du thermocouple et câble) est enfermée dans des gaines soudées sur les structures porteuses. Ces gainages peuvent contribuer à fausser légèroment les mesures, notamment lors des transitoires.

En outre, 65 thermocouples sont fixés sur la cuve principale en-dessous du niveau libre du sodium. Mais ils sont situés sur la paroi extérieure de la cuve, c'est-à-dire hors sodium.

\section{3 - Incertitude \\ des estimations faites en cours d'étude}

Cette incertitude est liée au caractère très tridimensionnel de l'espace annulaire d'intercuve traversé par six échangeurs, trois jupes de pompes, les tuyaux d'aspiration et le retour de la purification et l'excroissance, appelée «bec de cafetière », laissant passer la rampe de chargement-déchargement du combustible. Cette complexité géométrique rendait les calculs ardus.

Parmi les préoccupations du projeteur, on peut citer :

- La crainte de voir s'établir une «interface» brutale dans un plan horizontal entre le sodium froid du bas de l'intercuve et une couche de sodium chaud dans le haut de l'intercuve, sous le redan de la cuve primaire. Une telle interface, entre couche froide et couche chaude, aurait pu créer des gradients verticaux de température très élevés qui auraient causé des contraintes thermiques importantes dans les diverses viroles traversantes, notamment la virole inférieure de la cuve primaire et la cuve principale.

On trouve là, une des motivations ayant conduit à protéger la cuve principale (dont la bonne tenue est essentielle) par un circuit de refroidissement, utilisant la fuite aux pieds des assemblages, ménagé à l'aide du baffle et du contrebaffle.

- L'estimation de la fuite thermique, à travers la cuve primaire, du sodium chaud vers le sodium froid, en courtcircuitant les échangeurs chargés d'extraire la puissance du

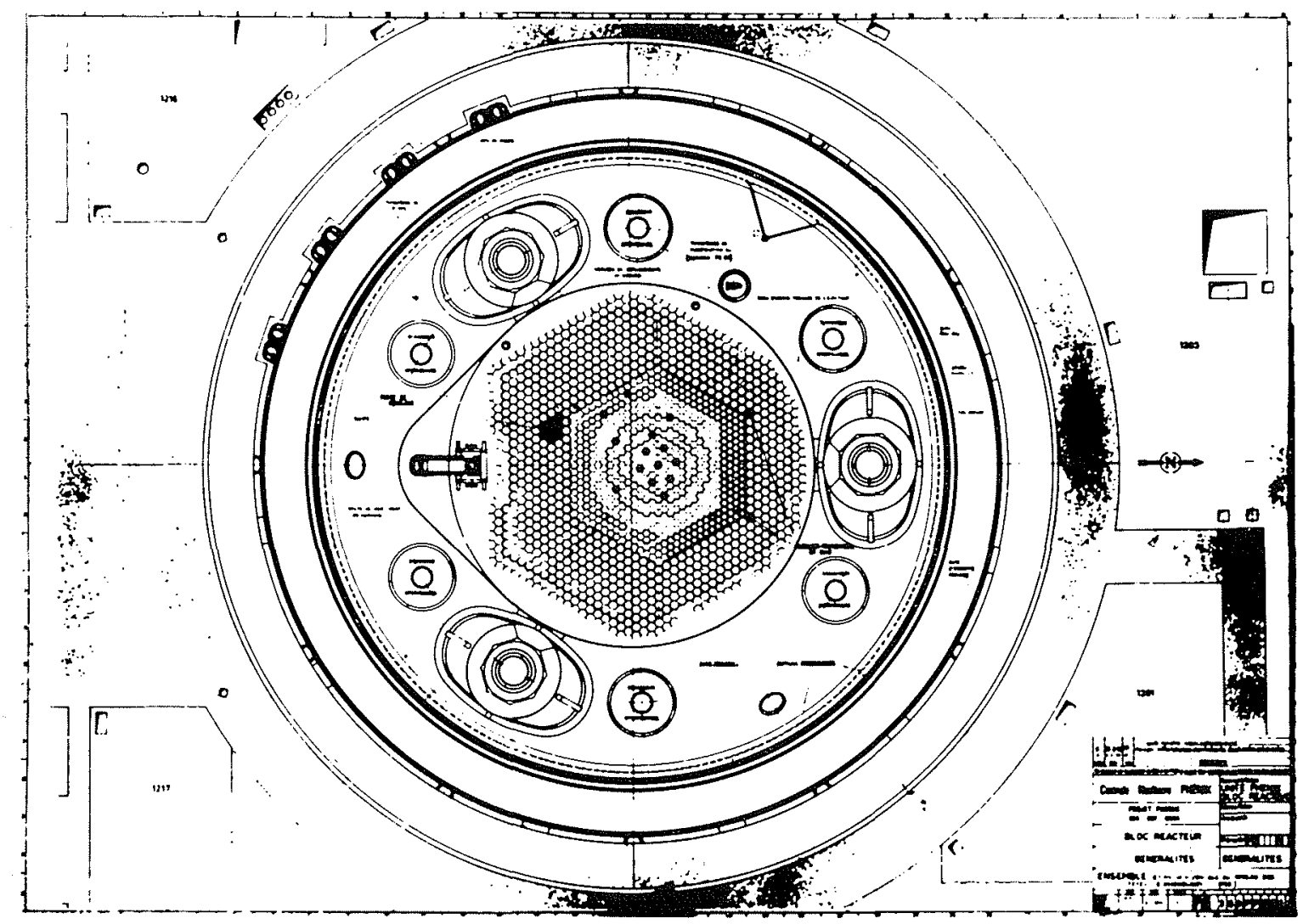


circuit primaire pour la transmettre au circuit secondaire. Par exemple, une intercuve rendue très froide jusque sous le redan de la cuve primaire, par des artifices utilisant l'effet de la convection forcée du sodium froid sortant des échangeurs, aurait augmenté cette fuite thermique. L'inconvénient est qu'une telle fuite trop importante pourrait réduire la puissance de l'installation, compte tenu des limitations sur la température maximale admissible sur la gaine des aiguilles combustibles.

\section{4 - Résultats des mesures effectuées au cours des essais de montée en puissance}

Parmi les résultats disponibles actuellement, c'est-à-dire jusqu'à $60 \%$ de la puissance nominale $P_{n}$ de l'installation (150 MWe et $340 \mathrm{MWth}$ ), nous avons choisi quelques cas de fonctionnement remarquables.

Ces résultats sont présentés sur des schémas présentant:

- à gauche, une coupe verticale de l'intercuve sur laquelle sont figurées les positions des thermocouples concernés;

- à droite, un graphique des répartitions de température sur les génératrices des structures instrumentées.

\section{1 - Palier à la puissance $10 \% \mathbf{P}_{n}$ environ (63 MWth) (Voir figure 3).}

Sur la coupe verticale, on trouve:

- les 8 thermocouples de la perche, entre protection neutronique latérale P.N.L. et cuve primaire;

- les positions de 12 thermocouples sur une génératrice de la cuve primaire;

- les 19 thermocouples de la perche d'intercuve;

- les 5 thermocouples du baffle-contrebafffe;

- les positions de 5 thermocouples sur une génératrice de la cuve principale.

La vitesse des pompes primaires est : $260 \mathrm{tr} / \mathrm{mn}$, donnant un débit de $3700 \mathrm{~m}^{3} / \mathrm{h}$.

On indique aussi :

- la température moyenne du sodium froid .. $367^{\circ} \mathrm{C}$;

- la température moyenne du sodium chaud . $425^{\circ} \mathrm{C}$.

On constate que :

a) Les 4 thermocouples inférieurs de la perche, entre P.N.L. et cuve primaire, sont plus froids que les thermocouples de même altitude de la cuve primaire. Celà traduit l'efficacité du déffecteur tronconique soudé sur la cuve primaire, au-dessus des têtes d'assemblage, pour empêcher la convection forcée d'entraîner du sodium chaud entre la P.N.L. et la cuve primaire. Et celà indique aussi que l'inentie thermique des rondins de la P.N.L. est importante, rendant l'échauffement de cette zone très lent.

b) Sur la génératrice de cuve primaire, une singularité apparaît au niveau de l'arrondi de raccordement supérieur du redan. Une explication satisfaisante à ce phénomène reste à trouver.

c) L'évolution de la température dans la masse de sodium de l'intercuve, donnée par les 19 thermocouples de la perche d'intercuve, est très progressive. Il ne se forme pas, à ce régime, d'interface brutale.

d) Les courbes de la cuve principale et du baffle montrent l'évolution de température du sodium du circuit de protection de la cuve principale.

\section{2 - Transitoire de l'état isotherme $250^{\circ} \mathrm{C}$ (manutention) à $10 \% P_{n}$}

Pour plus de clarté, nous avons cinq schémas montrant l'évolution en fonction du temps de la répartition de température sur les cinq structures :

Figure 4: Perche entre P.N.L. et cuve primaire.

On remarque bien que l'espace entre P.N.L. et cuve primaire reste froid, et que les températures y sont même en retard par rapport à la température moyenne du sodium froid.

Figure 5: Génératrice de cuve primaire.

On voit s'établir progressivement la répartition pour le régime $10 \% P_{n}$ décrite au paragraphe précédent, y compris la singularité au niveau de l'arrondi supérieur du redan.

Figure 6: Perche d'intercuve.

La pente de la courbe de répartition de température s'accroît progressivement au fur et à mesure que le $\Delta T$ (sortie-entrée cœur) s'accroît.

Figure 7 : Baffle et contrebaffe.

L'évolution est liée à celle de la température du sodium froid du circuit de protection de la cuve principale.

Figure 8: Cuve principale.

On note le retard thermique du thermocouple le plus élevé qui est situé au-dessus de la surface libre du sodium.

\section{3 - Palier à la puissance $60 \% P_{12}$ (150 MWe - 340 MWth)}

Cette figure 9 est analogue à la figure 3.

On peut noter que :

- la vitesse des pompes primaires est : $640 \mathrm{tr} / \mathrm{mn}$, donnant un débit de : $10500 \mathrm{~m}^{3 / \mathrm{h}}$;

- la température moyenne du sodium froid est: $380^{\circ} \mathrm{C}$;

- la température moyenne du sodium chaud est: $540^{\circ} \mathrm{C}$.

On a donc le même $\Delta T$ cœur $=160^{\circ} \mathrm{C}$ qu'au nominal, mais plus froid de $20^{\circ} \mathrm{C}$

On constate que :

- les phénomènes ne sont pas différents qualitativement de ceux constatés au palier $10 \% P_{n}$;

- la zone entre P.N.L. et cuve primaire reste froide;

- il n'y a pas de gradient sévère sur la cuve primaire; la singularité constatée sur l'arrondi supérieur du redan existe toujours;

- dans la masse de l'intercuve, l'évolution de la température reste progressive, mais la pente s'est accrue juste aut-dessus des fenêtres de sortie des échangeurs sans atteindre des valeurs sévères $\left(200^{\circ} \mathrm{C} / \mathrm{m}\right)$;

- l'accroissement du débit dans les baffles réduit la montée de température le long de la cuve principale. 

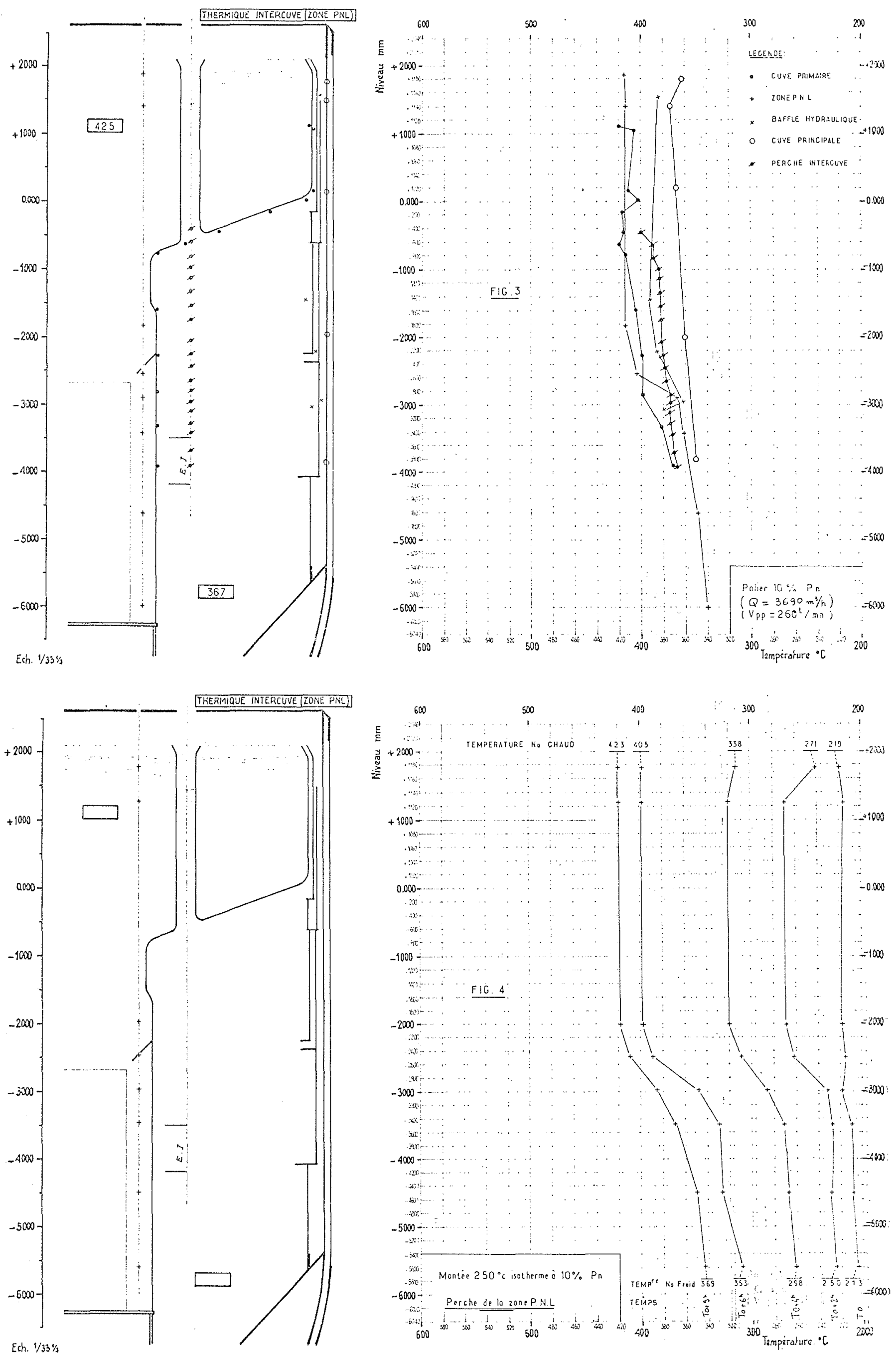

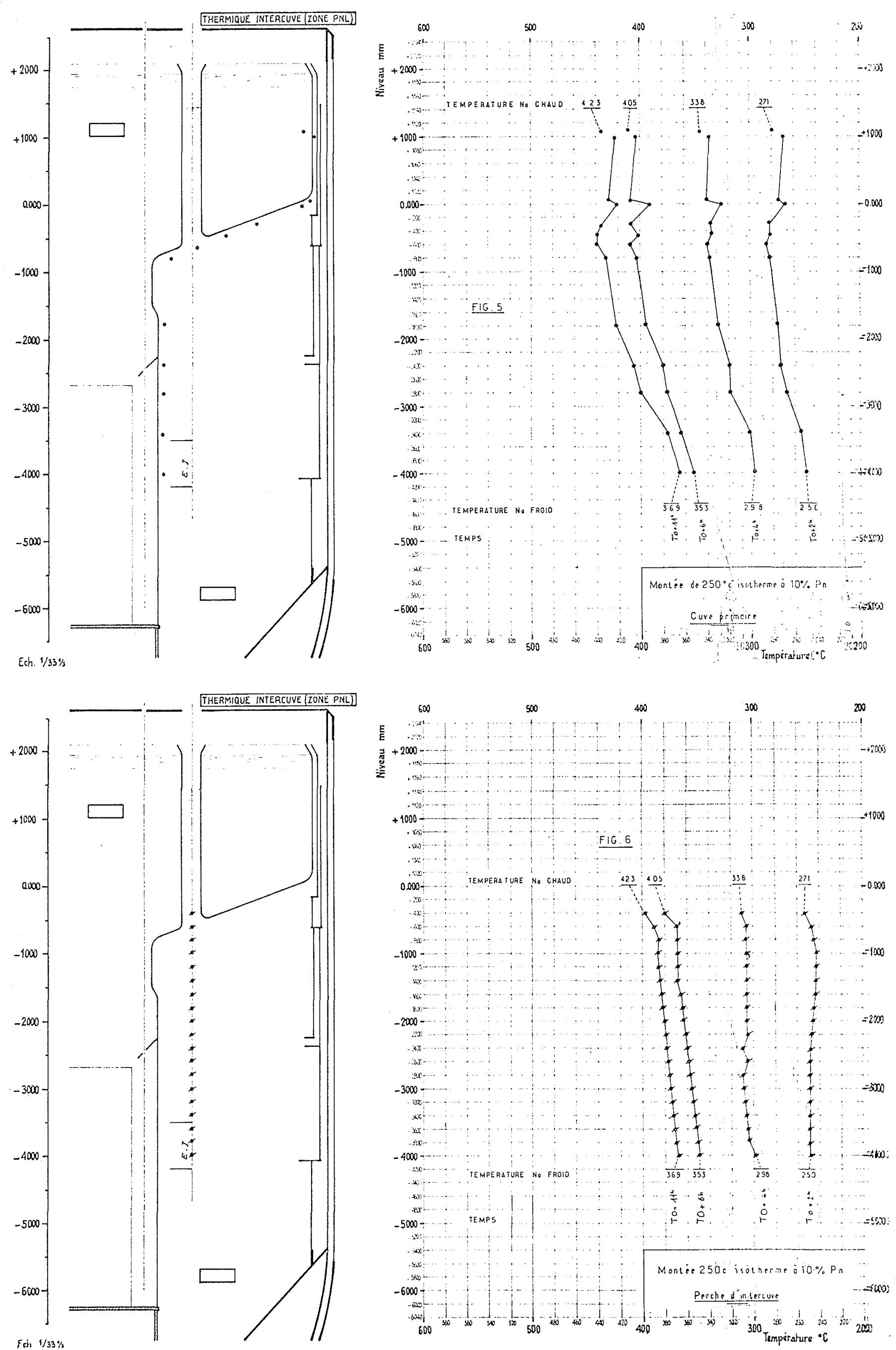

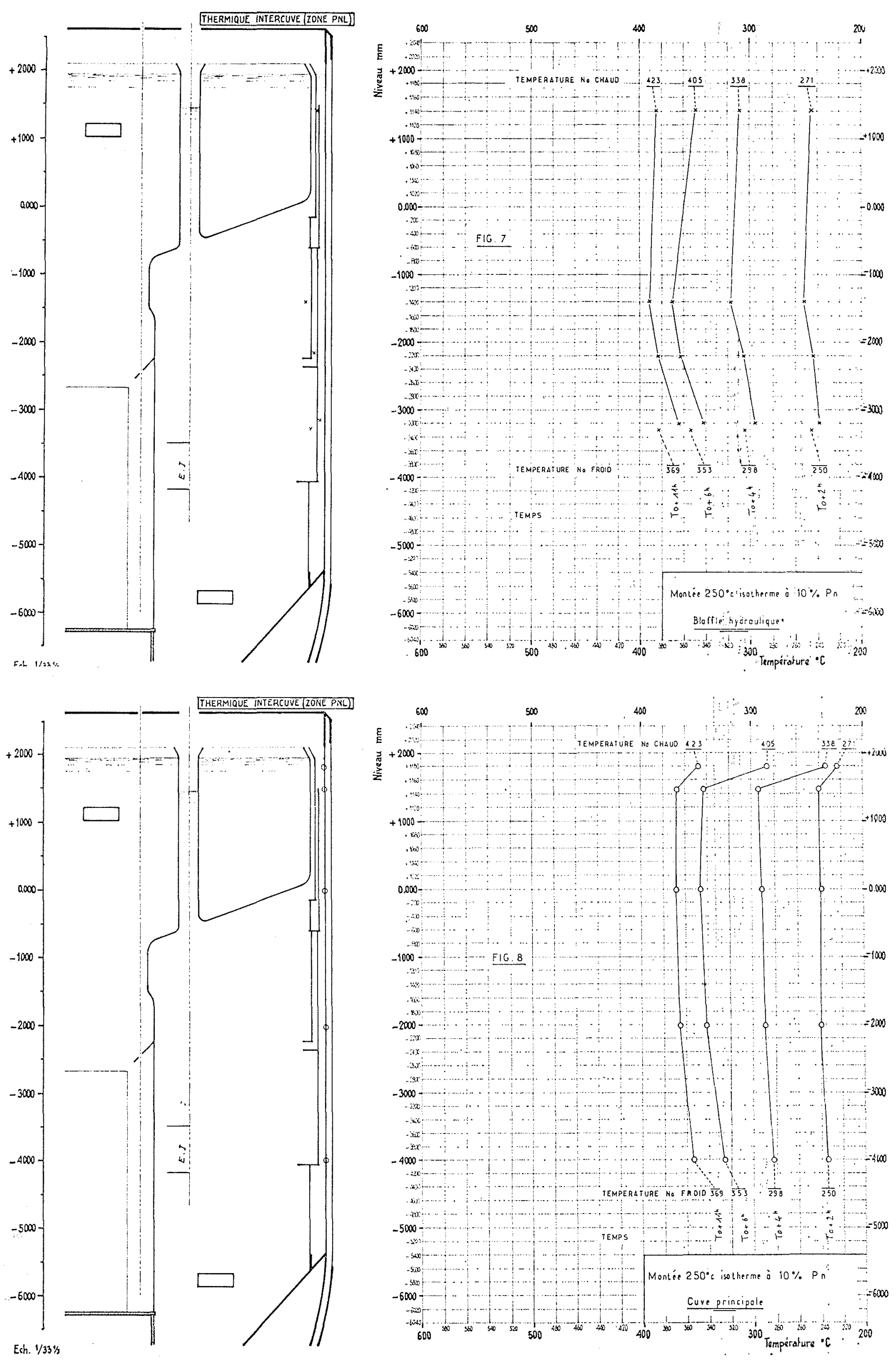

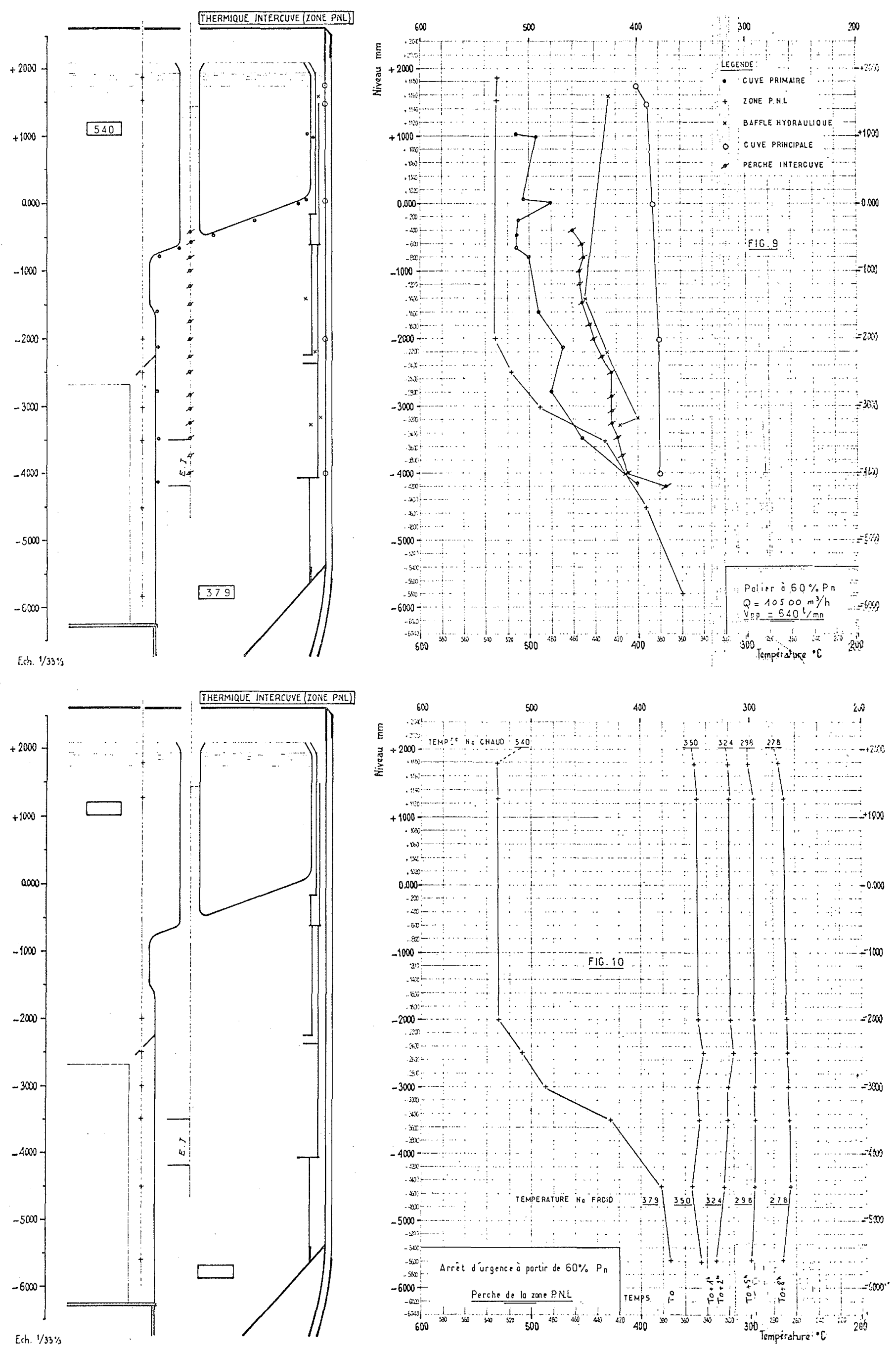

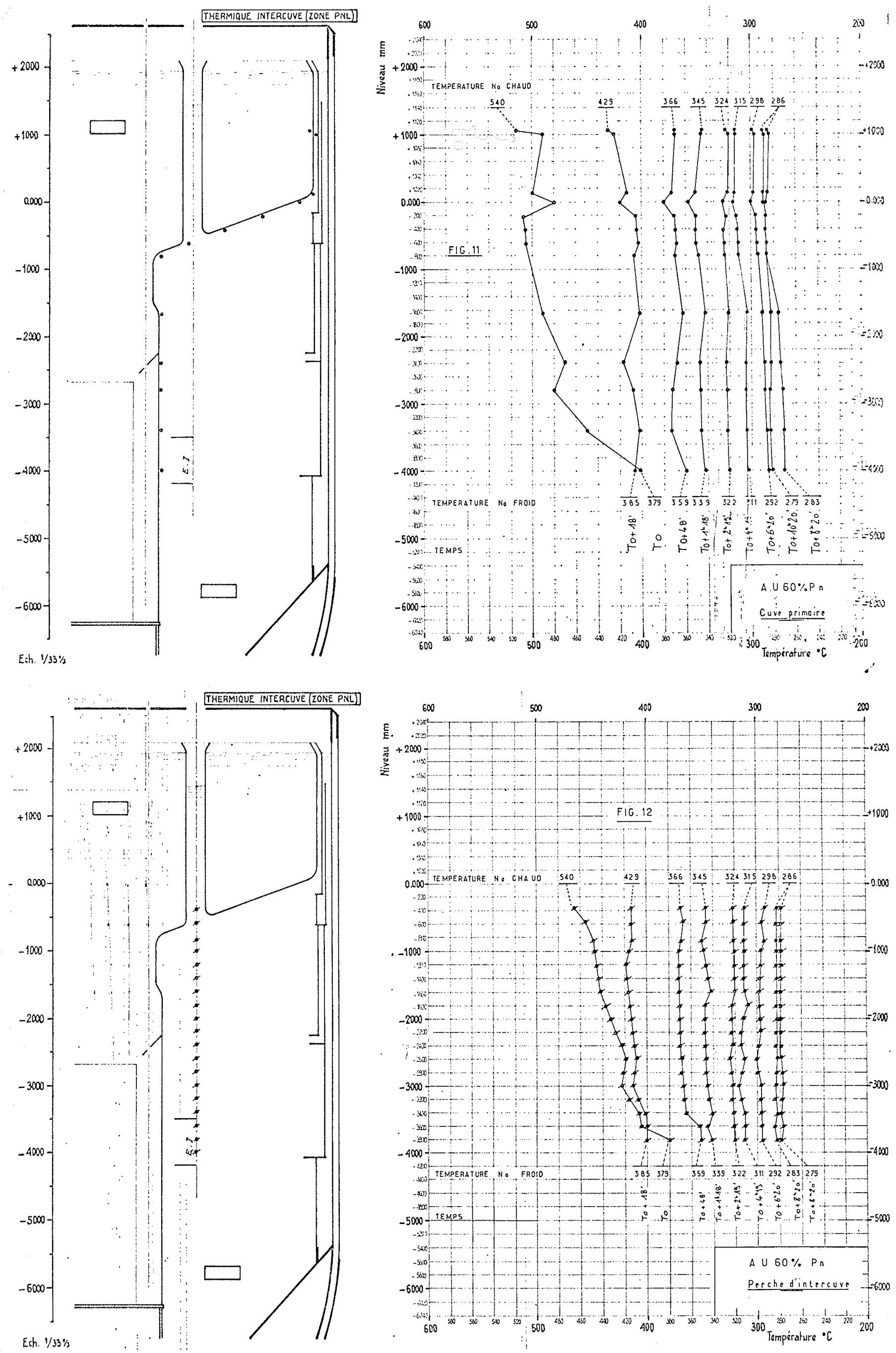

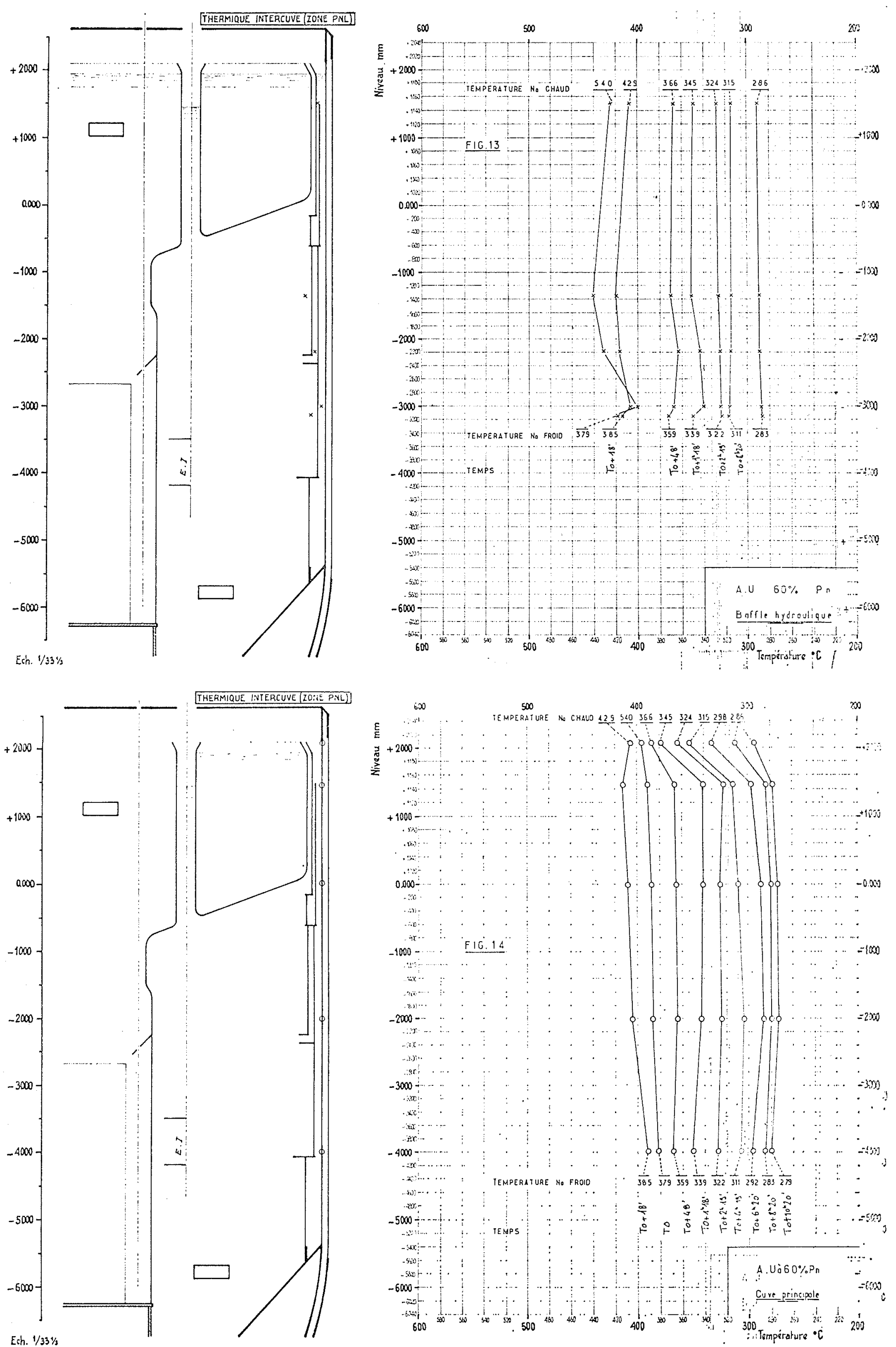


\section{4 - Arrêt d'urgence à partir du palier $60 \% P_{n}$}

Les figures $10,11,12,13$ et 14, pour l'arrêt d'urgence, sont homologues des figures $4,5,6,7$ et 8 pour la montée à $10 \% P_{n}$ à partir de l'état isotherme $250^{\circ} \mathrm{C}$.

Figure 10: Perche entre P.N.L. et cuve primaire.

On constate une homogénéisation des températures à l'intérieur de la cuve primaire. Puis, les indioations de tous les thermocouples baissent parallèlement.

Figure 11: Génératrice de cuve primaire.

L'évolution est similaire à celle de la figure précédente. On remarque une inversion de la singularité constatée au niveau de l'arrondi supérieur du redan. Cette singularité s'estompe vers la fin du transitoire où l'on atteint un état isotherme.

Figure 12: Perche d'intercuve.

Au temps $T_{0}+18 \mathrm{mn}$, on note une augmentation de la température indiquée par le thermocouple le plus bas, en face de la fenêtre de sortie des échangeurs $\mathrm{Ce}$ "choc chaud» est dû au ralentissement du débit secondaire.

Figure 13 : Baffle et contrebaffle.

Le phénomène signalé sur la figure 12 apparaît également.

Figure 14: Cuve principale.

Le «choc chaud» se propage dans le circuit de protection de la cuve principale, et se traduit par l'aug- mentation des températures du temps $T_{0}$ au tomps $T_{0}+18 \mathrm{mn}$. Par le thermocouple, situé au-dessus du niveau libre $\mathrm{du}$ sodium, on remarque le retard du refroidissement des structures en argon par rapport à celles en sodium.

\section{Conclusion}

Ces premiers relevés de mesures permettent de constater: - qu'il ne se produit pas d'interface brutale entre une couche froide et une couche chaude, capable d'induire des gradients importants et donc des contraintes élevées dans les structures;

- que les différences de température, de part et d'autre de la cuve primaire, ne sont pas très élevées, ce qui doit contribuer à rendre modérée la fuite thermique du sodium chaud vers le sodium froid; cette fuite thermique pourra être déterminée avec plus de précision à partir des bilans thermiques et neutroniques.

Il serait surprenant que le régime nominal caractérisé par les valeurs:

- vitesse des pompes primaires .... = $790 \mathrm{tr} / \mathrm{mn}$

- débit primaire ............. $=13000 \mathrm{~m}^{3 / \mathrm{h}}$

- température sodium chaud ....... $560{ }^{\circ} \mathrm{C}$

- température sodium froid ........ $400^{\circ} \mathrm{C}$

apporte des bouleversements par rapport à ces premières constatations, bien que les effets de la convection forcée se trouveront accentués.

\title{
Discussion
}

\author{
Président : M. H. MoNDIN
}

M. le Président remercie M. LALlement de son exposé et ouvre la discussion.

Celle-ci débute par un échange de vues entre M. le Président, M. le Professeur Fortier et M. Leduc sur les causes possibles de l'anomalie que présente la courbe des températures relevées au voisinage du redan au cours de différents essais.

Malgré diverses vérifications (et en particulier le réétalonnage du thermocouple intéressé), dit M. LALLEMENT, cette anomalie a subsisté et n'a pas encore trouvé d'explication.

Les mesures dans la masse du sodium de lintercuve sont assurées par des thermocouples montés sur une perche. Sur les parois de la cuve primaire, les thermocouples sont fixés sur des bouchons obturant des trous percés dans la tôle. L'extrémité de ces thermocouples se trouve dans un doigt de gant à $10 \mathrm{~mm}$ à l'extérieur de la cuve primaire, dans la couche limite du sodium. En principe, ces thermocouples mesurent la température du sodium dans l'intercuve.

M. le Président souligne le résultat remarquable des mesures que recoupent d'une manière satisfaisante les prévisions de nombreux calculs et essais. Il y a là un bel exemple de collaboration entre les diverses équipes qui ont concouru à cette importante réalisation.

Personne ne demandant la parole, M. le Président clôt cette première séance en remerciant tous les auteurs de communication et ceux qui, par leur intervention, ont rendu plus vivantes les discussions.

La séance est levée à $12 \mathrm{~h} 15$. 